\title{
An Empirical Model for Describing the Small Field Penumbra in Radiation Therapy
}

\author{
Shi-Qiang Tang, ${ }^{1}$ Yee-Min Jen, ${ }^{1,2}$ and Jia-Ming $W u\left(\mathbb{D}{ }^{1,2,3,4}\right.$ \\ ${ }^{1}$ Radiotherapy Center, Chenzhou No. 1 People's Hospital, Chenzhou, Hunan, China \\ ${ }^{2}$ Department of Radiation Oncology, Yee Zen General Hospital, Tao Yuan City, Taiwan \\ ${ }^{3}$ Department of Medical Physics, Chengde Medical University, Chengde City, Hebei Province, China \\ ${ }^{4}$ Department of Biomedicine Engineering, Chengde Medical University, Chengde City, Hebei Province, China \\ Correspondence should be addressed to Jia-Ming Wu; rtojmwu@gmail.com
}

Received 31 August 2019; Accepted 6 November 2019; Published 7 December 2019

Academic Editor: Yujiang Fang

Copyright (c) 2019 Shi-Qiang Tang et al. This is an open access article distributed under the Creative Commons Attribution License, which permits unrestricted use, distribution, and reproduction in any medium, provided the original work is properly cited.

\begin{abstract}
Purpose. We developed a mathematic empirical model for describing the small field penumbra in order to analyze the potential dose perturbation caused by overlapping field to avoid the dose calculation errors in linear accelerator-based radiosurgery. Materials and methods. A ball phantom was fabricated for measuring penumbra at 4 different gantry angles in the coplanar plane. A least square root estimation (LSRE) Model was created to fit the measured penumbra dose profile and to predict the penumbra dose profile at any gantry angles. The Sum of Squared Errors (SSE) was used for finding the parameters $n$ and $t$ for the best fitting of the LSRE model. Geometric and mathematical methods were used to derive the model parameters. Results. The results showed that the larger the gantry angle of the field, the more the expansion of the penumbra dose profile. The least square root estimation model for describing small field penumbra is as follows: Penumbra ${ }_{D(\check{s})}=T \cdot(1 / 2) \cdot\left(1-\left(\check{s} / \sqrt{\mathrm{n}+\check{s}^{2}}\right)\right)+\mathrm{t}$ where Penumbra ${ }_{D(\check{s})}$ denotes the dose profile $D(\tilde{s})$ at the penumbra region, $T$ is the penumbra height (usually in scalar 100), $n$ is the parameter for curvature, $\check{s}=x-W_{d} / 2$ ( $x$ and $\check{s}$ are the values in $\mathrm{cm}$ on $x$-axis), and $t$ is the radiation transmission of the collimator. Geometric analysis establishes the correlation between the penetration depth of the exposure and its effect on the penumbra region in ball phantom. The penumbra caused by an exposure at any arbitrary angles can be geometrically derived by using a one-variable quadratic equation. Conclusion. The dose distribution in penumbra region of small field can be created by the LSRE model and the potential overdosage or underdosage owing to overlapping field perturbation can be estimated.
\end{abstract}

\section{Introduction}

With the adoption of advanced technologies in modern radiotherapy such as stereotactic radiosurgery, stereotactic body radiation therapy, and intensity-modulated radiation therapy, there is an increased interest in the small-field dosimetry of photon beams. A beamlet used in linear accelerator-based radiosurgery is the smallest field formed by one single leaf of the MLC facing the opposite one and is only a portion of the target of dose delivery [1]. All these small fields are delivered and superimposed at different gantry angles during linear accelerator-based radiosurgery.
The analysis of small field penumbra is important in linear accelerator-based radiosurgery. It includes geometric, transmission, and photon scattered components. Geometric penumbra originates from the radiation source when it is not a single point. Transmission penumbra occurs when the beam passes through the edge of the jaw or MLC before it reaches the full attenuation point of the jaw and the MLC. Scattered radiation from the former two components is added to form the total penumbra, namely, the physical penumbra. The physical penumbra width is defined as the lateral distance from the central axis between $20 \%$ and $80 \%$ of the central axis dose at a reference depth. The height of a penumbra is defined as the intersection point of the central 
axis with the dose profile and is usually normalized to be $100 \%$.

We design a mathematical model to fit the penumbra in small field used in linear accelerator-based radiosurgery. The penumbra perturbation was also investigated by our model when one field is overlapped by another segment created by MLC. The dose perturbation at the field edges could lead to a potential monitor unit calculation error; therefore, this penumbra perturbation has to be accounted for in order to obtain the correct dose modification especially in the situation of split field [2].

\section{Materials and Methods}

2.1. Experiment Design and Steps. The experiment was conducted in the following steps:

(1) Establishment of a calibration curve for future dose quantification of irradiated films

(2) Single exposures of the standard reference field and with double exposure at four different gantry angles

(3) Establishment of a mathematical model, the least square root estimation (LSRE) model, and using the LSRE model to fit the penumbra dose profiles obtained in the previous step

(4) Using the Sum of Square Errors (SSE) to find the values of the LSRE model parameters

(5) Geometric derivation of the LSRE penumbra parameters at any gantry angle by using a transformation equation

(6) Estimating the dose curve expansion owing to overlapping field perturbation in split field by using LSRE

The details of each step are described in the following sections.

2.2. Establishment of a Calibration Curve. We used GAF Chromic EBT2 films (ISP Technology Inc., Wayne, NJ, USA; Lot \#F05090901) for the experiment. The film has a $Z_{\text {eff }}$ tissue equivalence of 6.98 with a fast polymerization in the imageforming process. Its absorption peaks are independent of radiation energy. Its higher sensitivity in the dose range of $2-800$ cGy and an inhomogeneity smaller than $2 \%$ after radiation exposure make it suitable for dosimetry analysis. The film processing and dose profile measurements followed the international protocols [3]. A preexposure technique was used for the calibration curve derivation [4]. This was performed by giving each film a priming dose of $2 \mathrm{~Gy}$ to homogenize the film density using an Elekta $6 \mathrm{MV}$ linear accelerator (Stockholm, Sweden) with a dose rate of $300 \mathrm{MU} / \mathrm{min}$. We measured the dose homogeneity using a densitometer. The previously exposed films were embedded into a solid water phantom at a depth of $5 \mathrm{~cm}$. Radiation was delivered with the same $6 \mathrm{MV}$ linear accelerator with a $10 \times 10 \mathrm{~cm}^{2}$ field size at an SAD of $100 \mathrm{~cm}$. Graded doses of 5 , $10,15,40,60,80,100,150$, and $200 \mathrm{cGy}$ were given to obtain the Hurter-Driffield calibration curve (H-D curve).
All exposed films were then scanned with an Epson Expression 10000XL scanner in the 48-bit RGB mode (16 bits per color), and the data were saved as tagged image file format (TIFF) and analyzed by the VariSoft imaging procession software. The films were scanned in the landscape orientation to reduce the optical density (OD) variation to $\leq 2 \%$ [5]. The experiments and film analysis were performed at room temperature to reduce the temperature-dependent evolution of the films [6]. All OD measurements were performed $24 \mathrm{~h}$ after irradiation to avoid time effect on the films. GAF chromic film was handled with forceps to avoid scratching and fingerprints.

The OD of the film was derived by the following equation:

$$
\mathrm{OD}=\log _{10} \frac{B_{0}}{B},
$$

where $B_{0}$ is the background density, namely, the scanner signal of the unexposed film, and $B$ is the scanner signal of the exposed film.

A red filter was placed on top of the GAF films before scanning to increase the slope of the H-D curve, thereby raising the resolution of the dose-OD curves [7].

\subsection{Single Exposures of the Standard Reference Field and with} Double Exposure at Four Different Gantry Angles. The single exposure of a radiation field $4 \times 2 \mathrm{~cm}^{2}$ on dimension $10 \times 10 \mathrm{~cm}^{2}$ GAF film was conducted in a regular $30 \times 30 \mathrm{~cm}^{2}$ polystyrene solid phantom at the depth of $5 \mathrm{~cm}$ with $100 \mathrm{cGy}$ as the standard reference of penumbra profile. The other four films with dimension $10 \times 10 \mathrm{~cm}^{2}$ were exposed each with a radiation field $4 \times 2 \mathrm{~cm}^{2}$ on GAF film in a regular $30 \times 30 \mathrm{~cm}^{2}$ polystyrene solid phantom at the depth of $5 \mathrm{~cm}$ with $100 \mathrm{cGy}$ as the first exposure and then follows the $6 \times 4 \mathrm{~cm}^{2}$ field at four different gantry angles in ball phantom separately. We chose a $4 \times 2 \mathrm{~cm}^{2}$ field for two reasons: firstly, to simulate the commonly used calculation grid size in the treatment planning systems; secondly, to mark dose profile measurement direction for a smaller filed size used in linear accelerator-based radiosurgery. The penumbra dose profile produced by this single exposure was used as the original standard reference data.

An acrylic ball phantom $22 \mathrm{~cm}$ in diameter was made for the second exposure. A wall laser was aimed at the ball equator plane to set the $\mathrm{SAD}$ at $100 \mathrm{~cm}$ isocentrically for the second exposure. The ball could be separated from its equator into two hemispheres. One of the two hemispheres has a $20 \times 20 \times 0.1 \mathrm{~cm}^{3}$ dent on the equator plane to hold the film (Figure 1). The four previously exposed $4 \times 2 \mathrm{~cm}^{2}$ films were put separately into the film holder on the equator plane for the second exposure with a $6 \times 4 \mathrm{~cm}^{2}$ field at $30^{\circ}, 45^{\circ}, 60^{\circ}$, and $90^{\circ}$ gantry angles, respectively (Figure 1 ).

The penetration depth remains the same at the $2 \mathrm{~cm}$ side on the $-y$ and $+y$ axis of the $4 \times 2 \mathrm{~cm}^{2}$ field at different gantry angles (Figure 1). On the other hand, the radiation penetration depth changes at the $4 \mathrm{~cm}$ side of the $4 \times 2 \mathrm{~cm}^{2}$ field on the axis of $-x$ and $+x$ from the larger second field. Because the gantry rotated around the $y$ axis on the $x z$ 


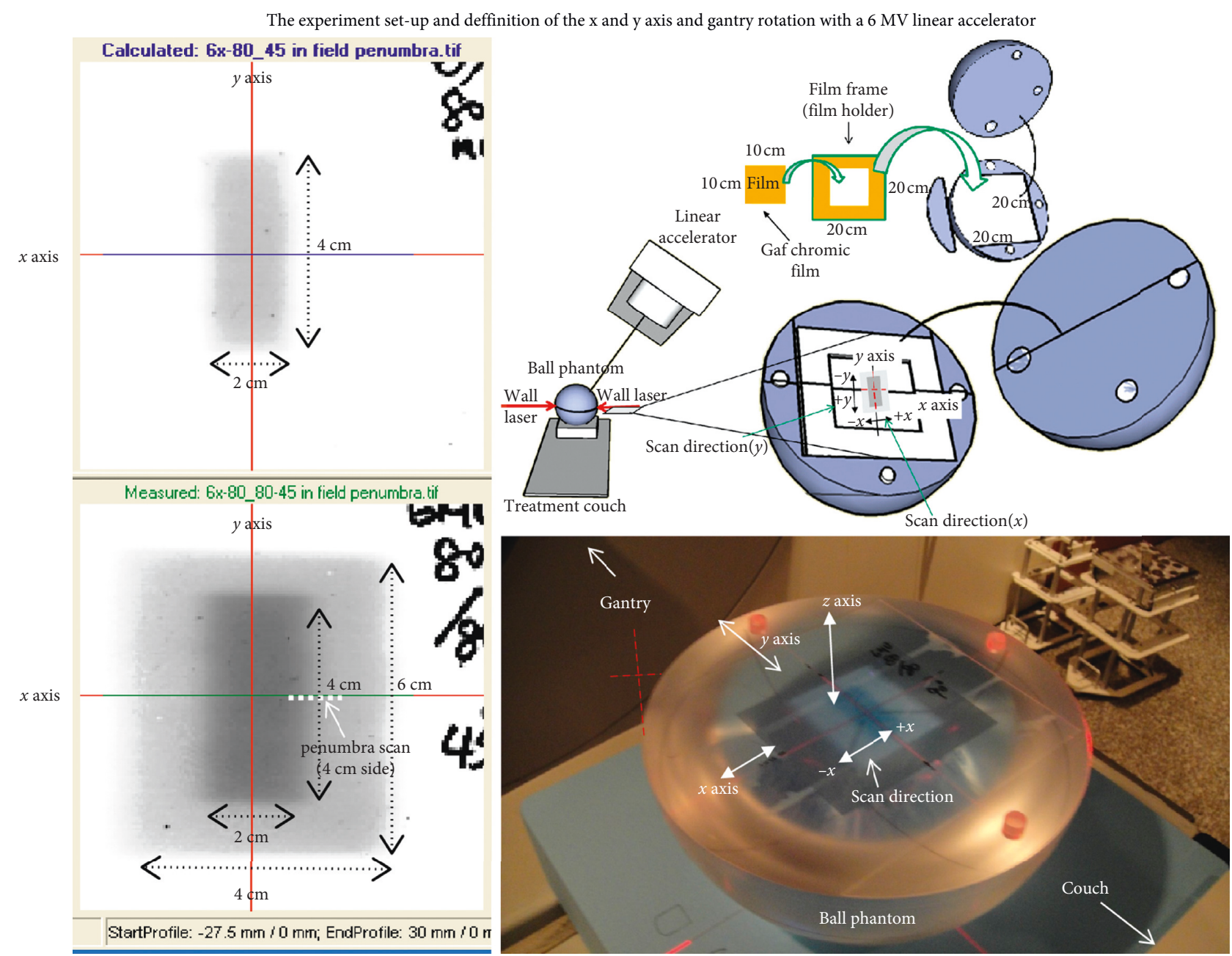

Figure 1: The custom-made ball phantom for the second film exposure. The $x, y$, and $z$ axes of the film are defined on this figure. The second exposure with a $6 \times 4 \mathrm{~cm}^{2}$ field was given to the film that was previously irradiated with a $4 \times 2 \mathrm{~cm}^{2}$ field. The film was embedded in the equator plane with the longitudinal direction parallel to the $y$ axis at different gantry angles for in-field penumbra perturbation study. Coplanar irradiation denotes that all the irradiations were delivered along the $x z$ axis with the same isocenter.

plane, the penetration depth from the larger second field changes only at the $4 \mathrm{~cm}$ side of the $4 \times 2 \mathrm{~cm}^{2}$ field on the axis of $-x$ and $+x$ (Figure 1). The penetration depth at the $2 \mathrm{~cm}$ side remains the same. This phenomenon led to different dose depositions on the penumbra region along the $4 \mathrm{~cm}$ side on the $-x$ and $+x$ axis compared to the $2 \mathrm{~cm}$ side on the $-y$ and $+y$ axis. We therefore analyzed only the $4 \mathrm{~cm}$ side.

2.4. Establishment of a Mathematical Model to Fit the Dose Profile of the $4 \times 2 \mathrm{~cm}^{2}$ Field after Superimposition by a $6 \times 4 \mathrm{~cm}^{2}$ Field at Different Gantry Angles in Coplanar Plane. A mathematical model, named the least square root estimation model (LSRE model), was developed for modeling the penumbra dose profile perturbation. The goal was to build a mathematical model which could predict the penumbra profiles at any gantry angles in coplanar irradiation. The LSRE model originated from the proportion function $y(x)=1 / x$. For details, please refer to Appendix A.
The least square root estimation model is expressed as follows:

$$
\operatorname{Penumbra}_{D(\check{s})}=T \cdot \frac{1}{2} \cdot\left(1-\frac{\check{s}}{\sqrt{n+\check{s}^{2}}}\right)+t,
$$

where Penumbra ${ }_{D(\check{s})}$ denotes the dose profile $D(\check{s})$ at the penumbra region; $T$ is the penumbra height representing the intersection of the beam central axis with the penumbra curve and is between 100 and $99.8 \% ; n$ is the parameter which gives the curve the curvature, and the greater the $n$ is, the flatter the penumbra would be; $\check{s}=x-W_{d} / 2$, where $\check{s}$ and $x$ are numbers on the $x$ axis in the unit of $\mathrm{cm}, W_{d} / 2$ is the half-field width or the width from the central axis to the $50 \%$ dose point of the penumbra region; and $t$ is the radiation transmission of the collimator.

The LSRE model was used to fit the penumbra of the single exposure standard reference curve and the $4 \times 2 \mathrm{~cm}^{2}$ dose profile on the $4 \mathrm{~cm}$ side after $6 \times 4 \mathrm{~cm}^{2}$ field double exposures at 4 different gantry angles in a coplanar 
irradiation. It fits the penumbra (Penumbra ${ }_{D(\check{s})}$ ) dose profile from the central axis to the end of the curve.

\subsection{Using the Sum of Square Errors (SSE) to Find the Optimaln} and $t$ of the LSRE Model. We need a tool to determine whether a best fit to the penumbra profile had been achieved or not by the LSRE. The Sum of Square Errors (SSE) provides an ideal measurement of the differences between the measured and calculated data by the LSRE model [8]. The lowest SSE indicates a minimum difference between the LSRE model prediction and the measured data.

The SSE is defined as follows:

$$
\mathrm{SSE}=\sum_{i=1}^{n}\left(x_{i}-\overline{x_{l}}\right)^{2},
$$

where $\left\{x_{i}\right\}$ is the observation and $\left\{\overline{x_{l}}\right\}$ is the means of predicted values.

The total SSE is

$$
\mathrm{SSE}_{\text {total }}=\mathrm{SSE}_{1}+\mathrm{SSE}_{2}+\mathrm{SSE}_{3}+\mathrm{SSE}_{4}+\cdots+\mathrm{SSE}_{n} \text {. }
$$

The $n$ that leads to the lowest SSE would result in the best fit of the penumbra curvature; the $t$ with the lowest SSE is derived separately and it affects the height of the penumbra tail.

2.6. Geometric Derivation of the Perturbation Effect on the Penumbra by a $6 \times 4 \mathrm{~cm}^{2}$ Second Field at Arbitrary Angles in Coplanar Irradiation. So far, we have the best fits of parameters $n$ and $t$ only for the dose profiles at gantry angles of $30^{\circ}, 45^{\circ}, 60^{\circ}$, and $90^{\circ}$. Eventually, we aim to obtain $n s$ and $t s$ at any gantry angles. For this, we need to find the lengths of $\overline{\mathrm{ed}}$ at a specific gantry angle because the parameters $n$ and $t$ were dependent only on the penetration depth $\overline{\mathrm{ed}}$. $\overline{\mathrm{ed}}$ can be derived by using geometry (Figure $2(\mathrm{a})$ ). $\overline{\text { ed }}$ is the penetration length on the $\overline{S / c}$ of the larger field at a gantry angle of $\theta$. It is the distance where the radiation traverses inside the phantom before intersecting with the penumbra edge of the previous field. It is the only factor controlling the dose contribution from the larger superimposing field onto the smaller field along $\overline{\mathrm{od}}$. The larger $\overline{\mathrm{ed}}$ is, the more the attenuation of the passing radiation will be with less contribution to the penumbra. ed is also the penetration depth on the $\overline{\mathrm{S} / \mathrm{C}}$ of the $6 \times 4 \mathrm{~cm}^{2}$ field. $\overline{\mathrm{S} / \mathrm{C}}$ is the radiation line within the penumbra region from the source $S^{\prime}$ in the larger field along the $+x$ and $-x$ at an arbitrary gantry angle of $\theta$. For the detailed geometric derivation of $\overline{\mathrm{ed}}$, please refer to Figure 2(b) and Appendix B.

\section{Results}

3.1. Calibration Curve. When the film was scanned with a red color filter, the calibration curve was steeper and thus with a higher OD-dose resolution. Radiation dose was then derived by the OD method (please refer to Figure 3 ).

3.2. The Dose Profile of the $4 \times 2 \mathrm{~cm}^{2}$ Field with a Second Exposure by a $6 \times 4 \mathrm{~cm}^{2}$ Field at 4 Different Gantry Angles in
Coplanar Irradiation. Figure 4 demonstrates the single exposure as the standard reference penumbra profile and the cumulative dose profiles after double exposures on $4 \times 2 \mathrm{~cm}^{2}$ with a $6 \times 4 \mathrm{~cm}^{2}$ field at 4 different gantry angles. The dose profiles on the + axis are slightly higher than the dose profile on the $-x$ side. This is caused by smaller penetration depths $\overline{\mathrm{ed}} \mathrm{s}$ on the $+x$ axis than $\overline{\mathrm{e}^{\prime} \mathrm{d}^{\prime}} \mathrm{s}$ at the $-x$ side in the ball phantom (Figure 2(a)). The Full-Width Half Maxima (FWHM, the width between $50 \%$ dose of the $-x$ axis and the $+x$ axis) was $19.8 \mathrm{~mm}$ after single irradiation at a gantry angle of 0 in the flat polystyrene solid phantom. The FWHM became $20.3 \mathrm{~mm}, 21.1 \mathrm{~mm}, 21.3 \mathrm{~mm}$, and $21.6 \mathrm{~mm}$ after second exposures at gantry angles of $30^{\circ}, 45^{\circ}$, $60^{\circ}$, and $90^{\circ}$ in the ball phantom, respectively (Figure 4 ). The penumbra width between $80 \%$ to $20 \%$ of the central axis was $2.8 \mathrm{~mm}$ after a single exposure at $0^{\circ}$ with a $4 \times 2 \mathrm{~cm}^{2}$ field (Figure 4 ). After the second exposure, they were $4.7 \mathrm{~mm}, 6.2 \mathrm{~mm}, 6.7 \mathrm{~mm}$, and $8.1 \mathrm{~mm}$ at gantry angles of $30^{\circ}, 45^{\circ}, 60^{\circ}$, and $90^{\circ}$, respectively (Figure 4). The renormalized dose profile shows, as expected, no change of the penumbra width after double exposures of any doses with a $6 \times 4 \mathrm{~cm}^{2}$ field as long as the exposures were delivered at the same gantry angle as the first. The dose profile changes only after double exposures delivered at different gantry angles.

3.3. Dose Profile Fitting Using the LSRE Model. Parameters $T$, $n$, and $t$ in the LSRE model determine the dose profile shape (equation (2)). The right-side dose profile after the first exposure at a gantry angle of $0^{\circ}$ can be fitted accurately with $T=99.8, n=90$, and $t=0.2$ (Figure 5(a)). $n$ and $t$ that result in the best fit, denoted as $(n, t)$, at gantry angles of $30^{\circ}, 45^{\circ}, 60^{\circ}$, and $90^{\circ}$, are $(310,2),(410,3),(490,4)$, and $(650,5)$, respectively.

The measured curves of the left-side dose region can also be estimated by adjusting the $n, \mathrm{~T}$, and $t$ (Figure 5(b)). Parameters $n$ and $t$ of the left-side curve at 0 gantry angle after single exposure were $(90,0.2)$. After the second exposures, the parameters $(n, t)$ were $(320,1.8),(430,2.8),(510$, $3.6)$, and $(700,4.8)$ at gantry angles of $30^{\circ}, 45^{\circ}, 60^{\circ}$, and $90^{\circ}$, respectively.

3.4. Sum of Square Errors (SSE): Finding the Best $n$ and $t$. Figures $6(\mathrm{a})$ and $6(\mathrm{~b})$ show $n$ and $t$ with the smallest SSE which gives the best LSRE model fitting of the measured data at the gantry angle of $45^{\circ}$. The best fit at other gantry angles can be done in the same manner.

3.5. Geometric Derivation of the Effects on the Penumbra by Second Exposures at Arbitrary Gantry Angles. We found that the relationship of $e^{-\mu \omega}$ with the $x$ is a straight line (Figures $7(\mathrm{a})$ and $7(\mathrm{~b})$ and Table 1 ), where $\omega$ denotes the penetration depth $\overline{\mathrm{ed}}, \mu$ is the attenuation coefficient, and $\mu=0.00494 \mathrm{~cm}^{-1}$ of $6 \mathrm{MV}$ in water. The linear regression of $\mathrm{e}^{-\mu \omega}$ versus $x$ at different gantry angles can be described in the form of $y=u x+v$, where $u$ is the slope of the regression line shown in Figures $7(\mathrm{a})$ and 7(b). Figure 7(a) 


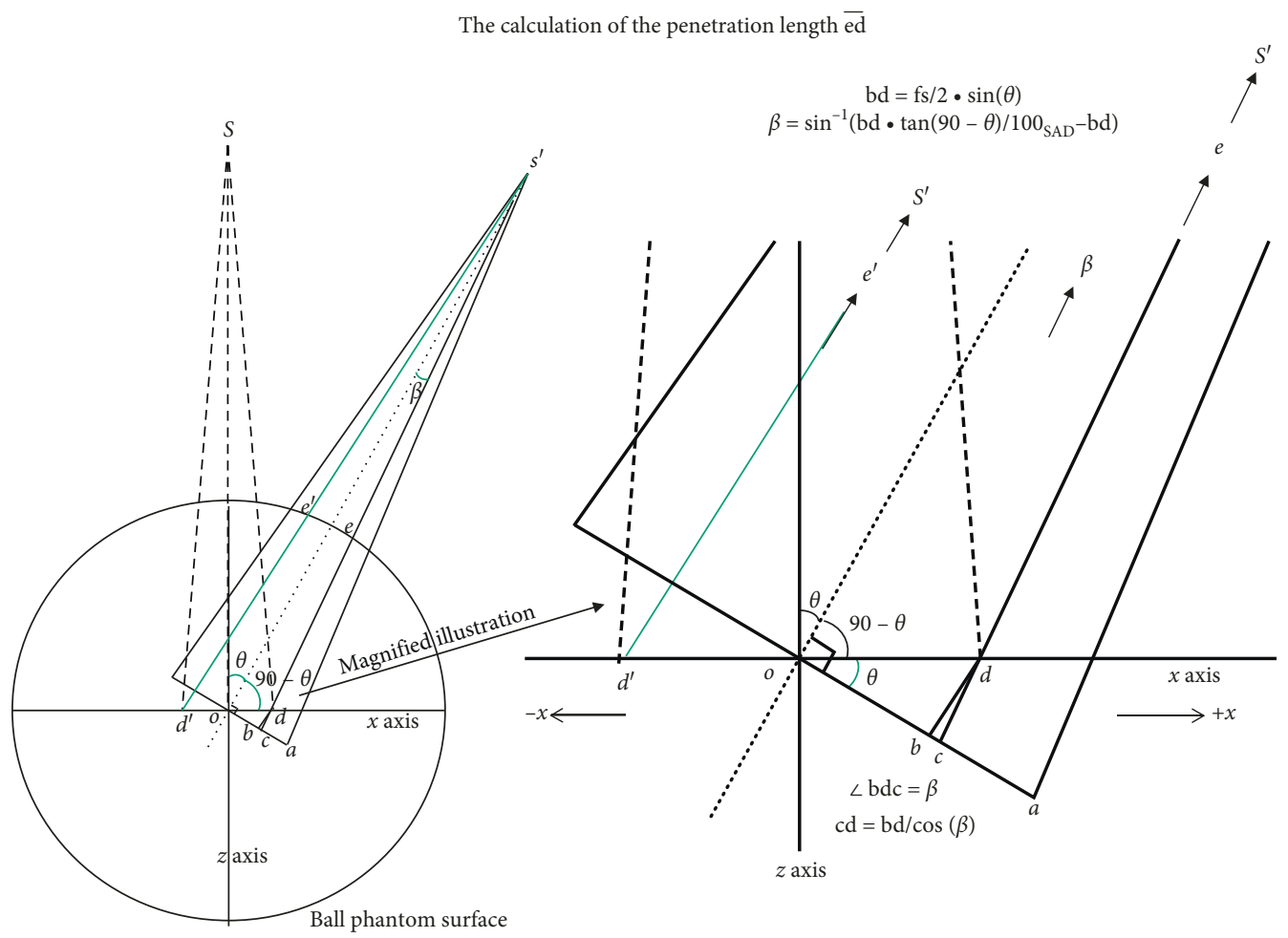

(a)

Derivation of the penetration length $\overline{\mathrm{ed}}$

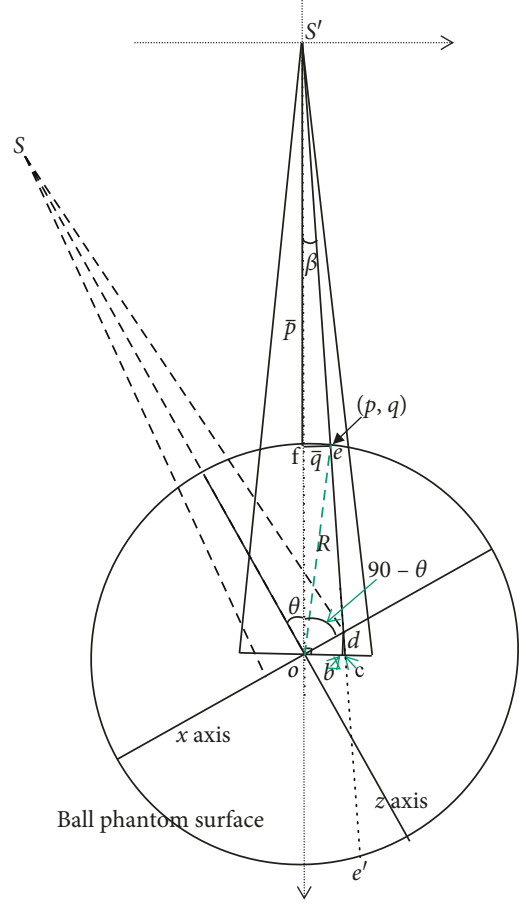

(b)

Figure 2: (a) The calculation of the length $\overline{e d}$ at a gantry angle of $\theta$ on $\overline{s^{\prime} \mathrm{c}}$ by geometric method after double exposures with a larger and a smaller field. The larger field was $6 \times 4 \mathrm{~cm}^{2}$ and the smaller field was $4 \times 2 \mathrm{~cm}^{2}$ field, respectively. $S$ represents the linear accelerator radiation source position with a gantry angle of $0^{\circ}$, and $S^{\prime}$ denotes the source position after a gantry rotation of $\theta$ degrees. $o$ is the rotational isocenter; $d$ is the field edge of the small field delivered at $0^{\circ}$ at the $4 \mathrm{~cm}$ side of the $4 \times 2 \mathrm{~cm}^{2}$ field; $a$ indicates the field edge of the larger field at the $6 \mathrm{~cm}$ side of the $6 \times 4 \mathrm{~cm}^{2}$ field when the gantry rotates to the angle of $\theta$. od is the half-field size of the smaller field on the $4 \mathrm{~cm}$ side of the $4 \times 2 \mathrm{~cm}^{2}$ field while $\overline{\mathrm{oa}}$ is the half-field size of the larger field on the $6 \mathrm{~cm}$ side of the $6 \times 4 \mathrm{~cm}^{2}$ field. fs $/ 2$ denotes the half-field size on the $4 \mathrm{~cm}$ side of the $4 \times 2 \mathrm{~cm}^{2}$ field $(\overline{\mathrm{od}})$ and FS/2 denotes the half-field size on the $6 \mathrm{~cm}$ side of the $6 \times 4 \mathrm{~cm}^{2}$ field (FS/2). (b) This figure shows the geometric derivation of ed. $\overline{\text { ed }}$ is the key factor controlling the dose distribution from the larger field onto the penumbra region of the smaller field along $\overline{\mathrm{od}}$. $\overline{\mathrm{ed}}=\overline{\mathrm{S}^{\prime} \mathrm{c}}-\overline{\mathrm{cd}}-\overline{\mathrm{S}^{\prime} \mathrm{e}}$, where $\overline{\mathrm{S}^{\prime} \mathrm{c}}=100 / \cos (\beta)$, and $\overline{\mathrm{cd}}=\overline{\mathrm{bd}} / \cos (\beta)$. For details, please refer to Appendix B. 


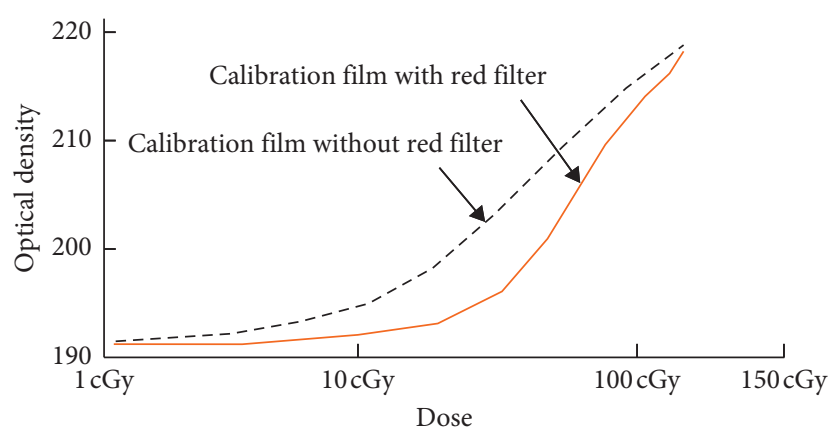

FIgURE 3: The calibration curve was steeper and thus more sensitive when the film was scanned with a red color filter.

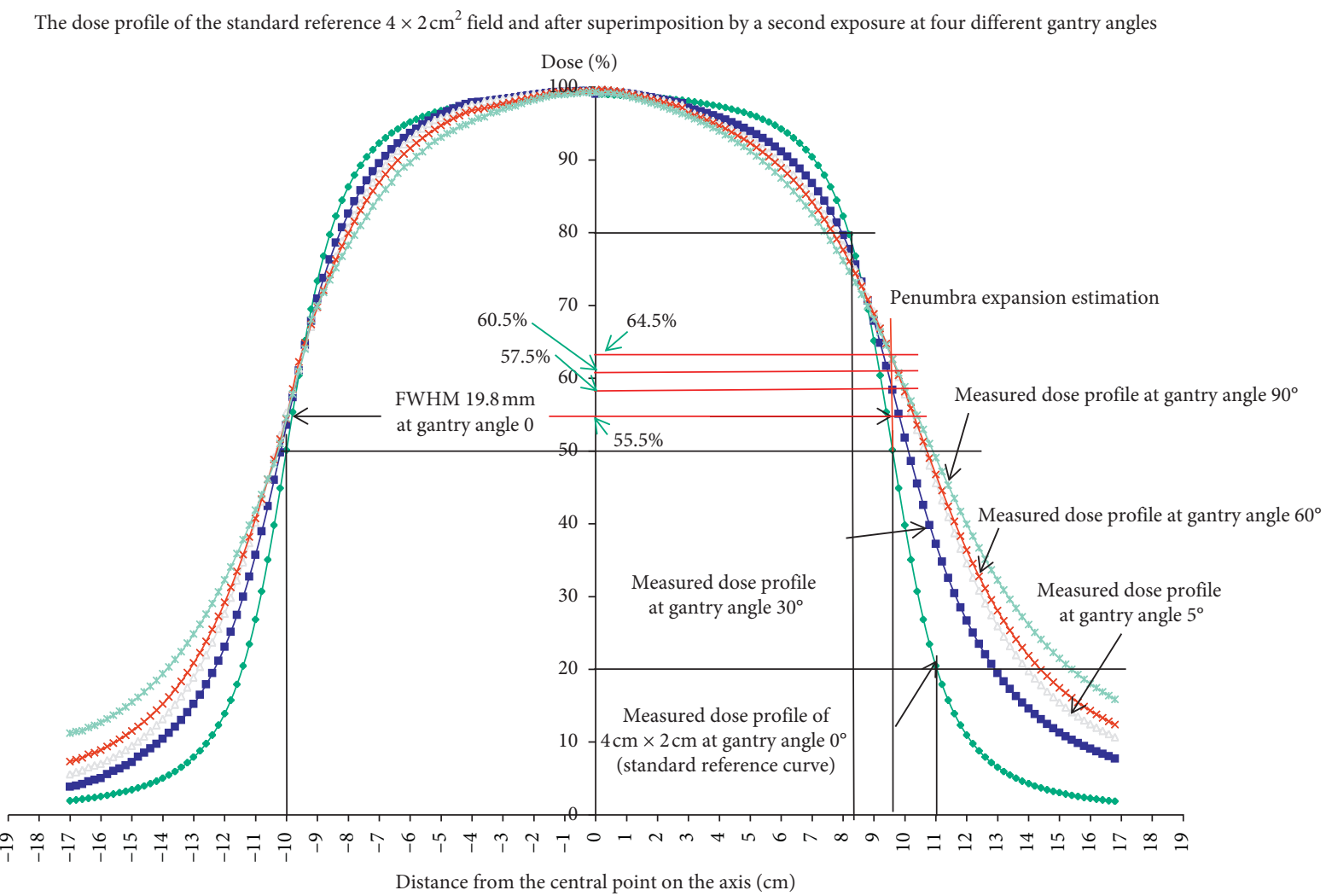

Figure 4: The dose profile of the smaller $4 \times 2 \mathrm{~cm}^{2}$ field irradiated at a gantry angle of $0^{\circ}$ at the flat polystyrene solid phantom, followed by a second exposure in the ball phantom with a larger field of $6 \times 4 \mathrm{~cm}^{2}$ at different gantry angles. The dose profile changes after second exposures at a gantry angle different from the first exposure. It also shows the full-width half maximum (the width at $50 \%$ dose of the $-x$ axis and the $+x$ axis) and the penumbra width from $80 \%$ to $20 \%$ of the central axis.

demonstrates the 4 lines with their slopes at the gantry angles of $30^{\circ}, 45^{\circ}, 60^{\circ}$, and $90^{\circ}$. The slopes at any other gantry angles can be derived by interpolation from these 4 know angles.

\subsection{Derivation of the Parameters $n$ and tof the Penumbra Dose} Profiles at Arbitrary Gantry Angles Using Mathematical Methods. $\overline{\mathrm{ed}}=\overline{\mathrm{S}^{\prime} \mathrm{c}}-\overline{\mathrm{cd}}-\overline{\mathrm{S}^{\prime} \mathrm{e}}$, where $\overline{\mathrm{S}^{\prime} \mathrm{c}}=100 / \cos (\beta), \overline{\mathrm{cd}}=$ $\overline{\mathrm{bd}} / \cos (\beta)$, and

$\overline{\text { ed }}$ is the penetration depth of the $\overline{S^{\prime} c}$ from the larger field in the ball phantom. $\mathrm{S}^{\prime} \mathrm{e}$ can be derived from equations (5)-(7) (for details, please refer to Appendix B).

$$
\begin{gathered}
p^{2}-2 \mathrm{sp}+s^{2}+q^{2}=R^{2} \\
p=\frac{2 s \pm \sqrt{4 s^{2}-4\left(1+\xi^{2}\right)\left(s^{2}-R^{2}\right)}}{2\left(1+\xi^{2}\right)} \\
\overline{S^{\prime} \mathrm{e}}=\sqrt{p^{2}+q^{2}}=\sqrt{\left(1+\xi^{2}\right) p^{2}}=\frac{s-\sqrt{s^{2}-\left(1+\xi^{2}\right)\left(s^{2}-R^{2}\right)}}{\sqrt{\left(1+\xi^{2}\right)}} .
\end{gathered}
$$

Table 1 shows the calculated $\overline{\text { ed }}$ s in Figure $2(\mathrm{~b})$ in the ball phantom at different gantry angles in coplanar irradiation. 


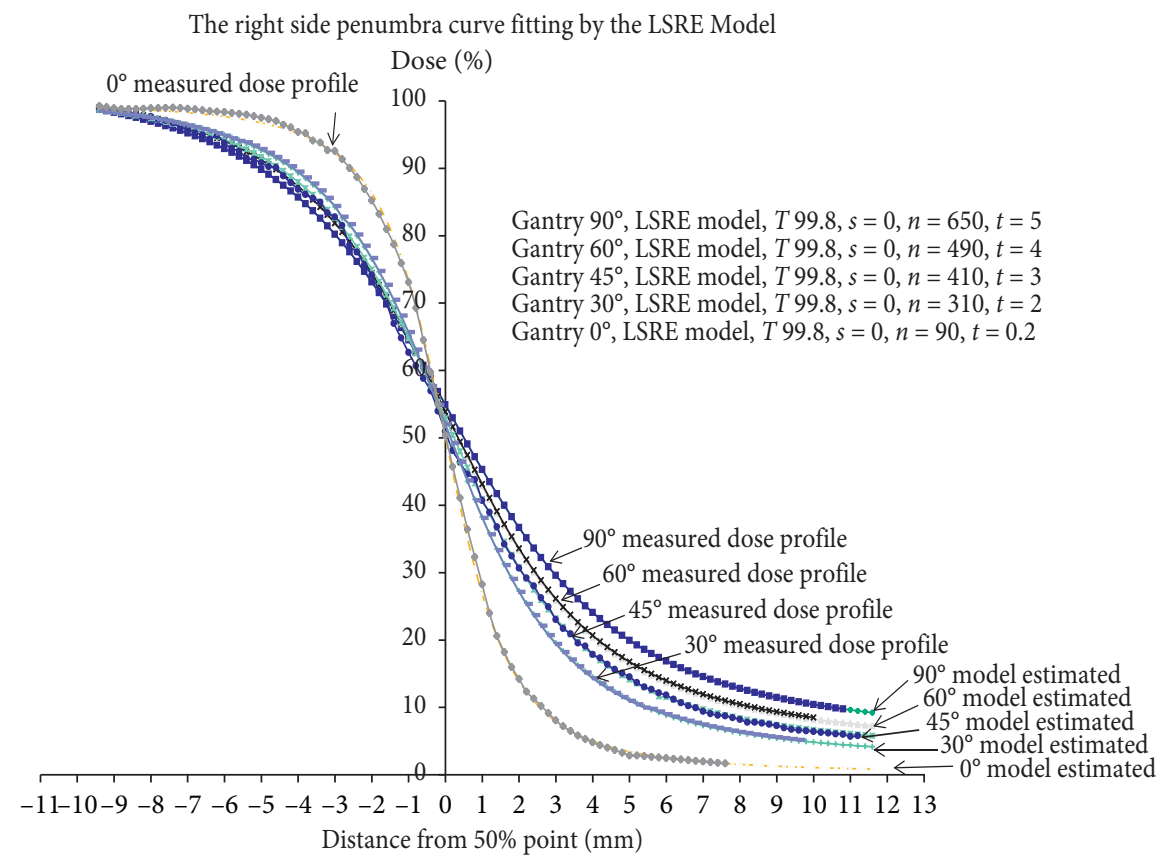

(a)

The left side penumbra curve fitting by the LSRE Model

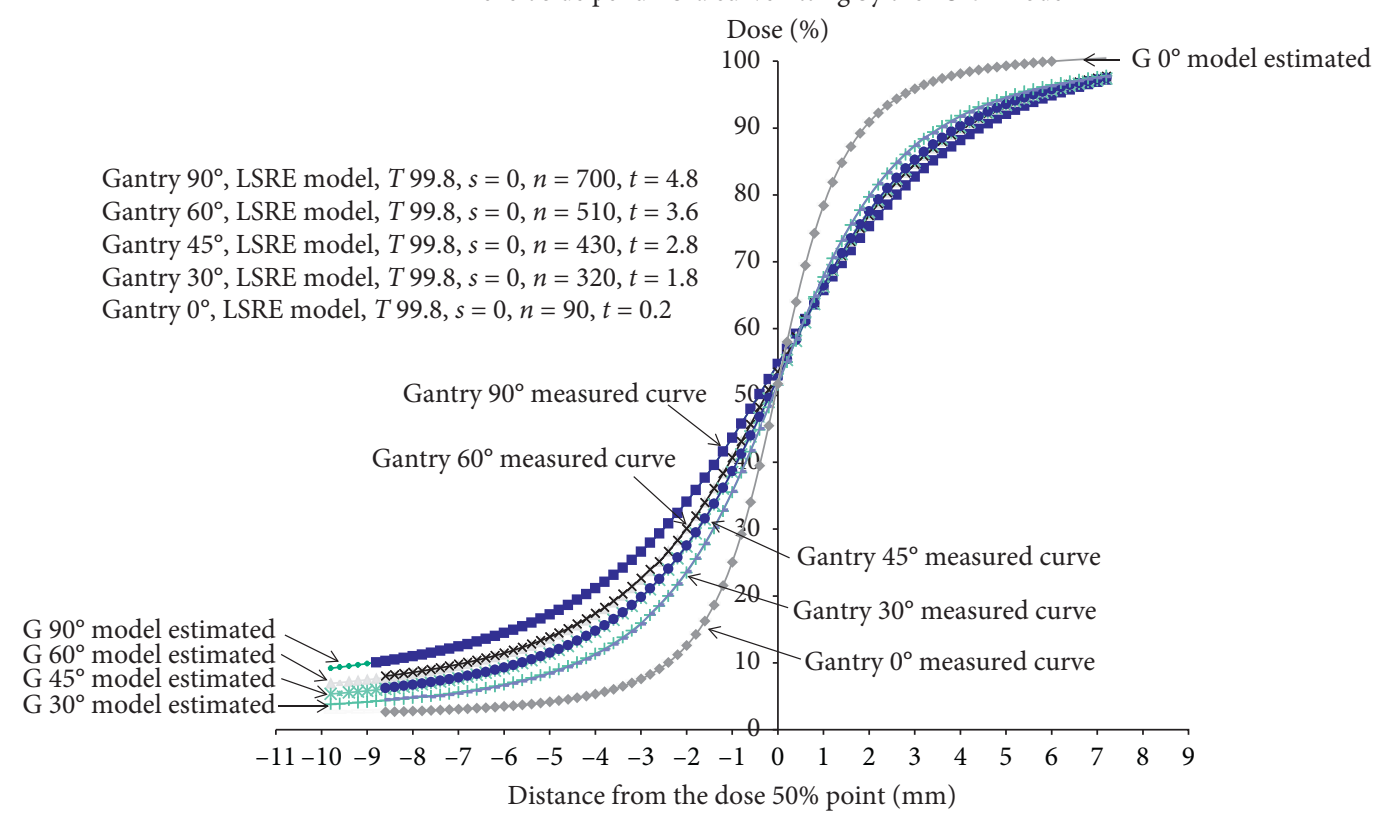

(b)

FIGURE 5: (a) The right-side penumbra dose profiles vary with the gantry angles of the second exposure. The curvature of the right-side penumbra region can be fitted using the LSRE model by adjusting the $(n) T$ and $(t)$. (b) The left-side penumbra dose profiles vary with the gantry angles of the second exposure. The curvature of the left-side penumbra region can be fitted using the LSRE model by adjusting the ( $n$ ) $T$ and $(t)$. The " $90^{\circ}$ measured curve" and " $90^{\circ}$ model estimated" curve denote the dose profile measured at the gantry angle of $90^{\circ}$ in the ball phantom and the predicted curve at the same. They overlap each other completely.

The regression curves for slopes versus $n$ and $t$ were shown in Figures 8(a) and 8(b), respectively. Therefore, once we obtain the slope of a regression curve from Figures $7(\mathrm{a})$ and $7(\mathrm{~b}), n$ and $t$ can be found in Figures 8(a) and 8(b). This enables us to derive the estimated curve with precision and thus the accurate effects on the penumbra of a second irradiation at any gantry angles without the labor conducting the actual experiment.

\section{Discussion}

We developed the LSRE model for studying the penumbra perturbation incurred by field overlapping in IMRT. This model has several advantages for penumbra profile fitting. Several functions, such as cosine [9] and exponential [10] functions, have been used in commercially available 
SSE curve of parameter $n$

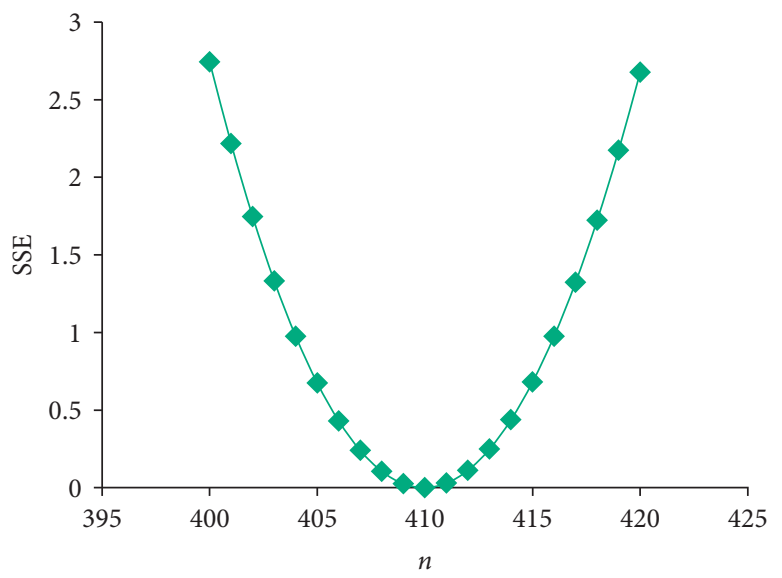

(a)

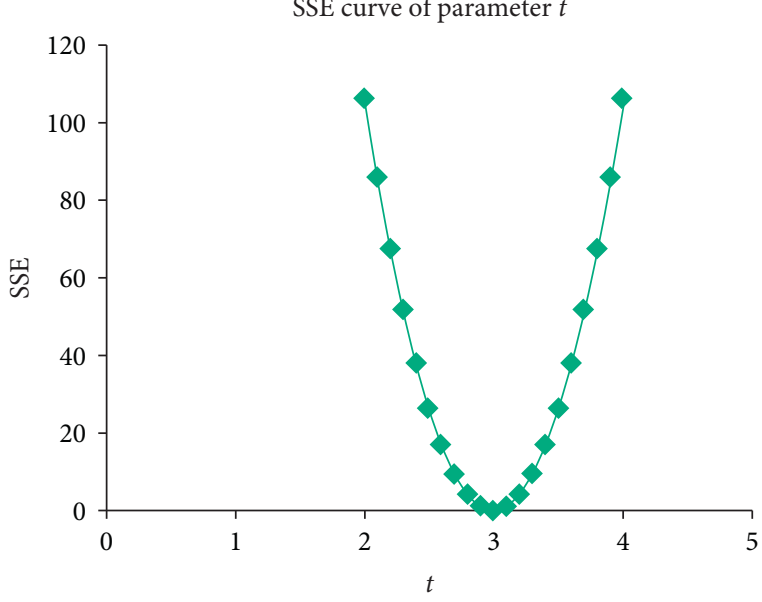

(b)

Figure 6: $(\mathrm{a}, \mathrm{b})$ These figures demonstrate the use of the SSE to find the best $n$ and $t$ of the LSRE model at the gantry angle of $45^{\circ}$.

The regression lines of $e^{-\mu \omega}$ versus $+x$ at different gantry angles

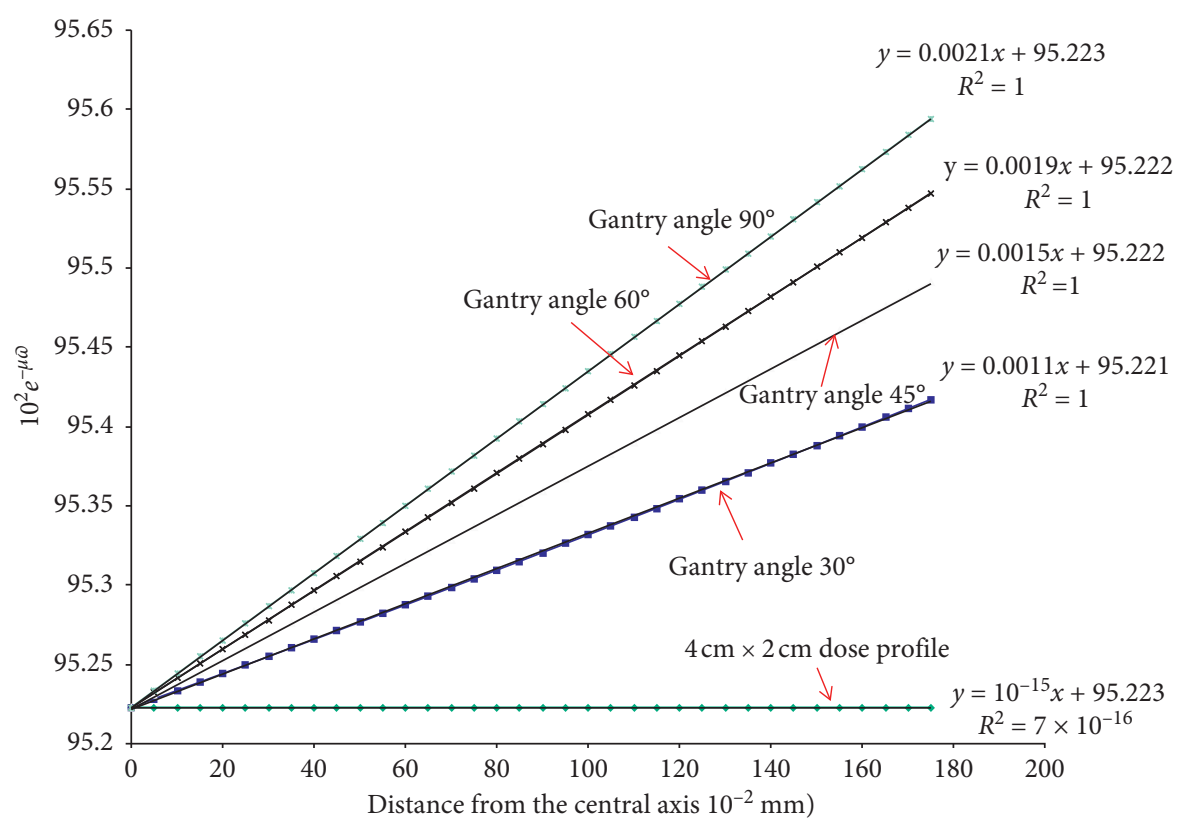

(a)

Figure 7: Continued. 


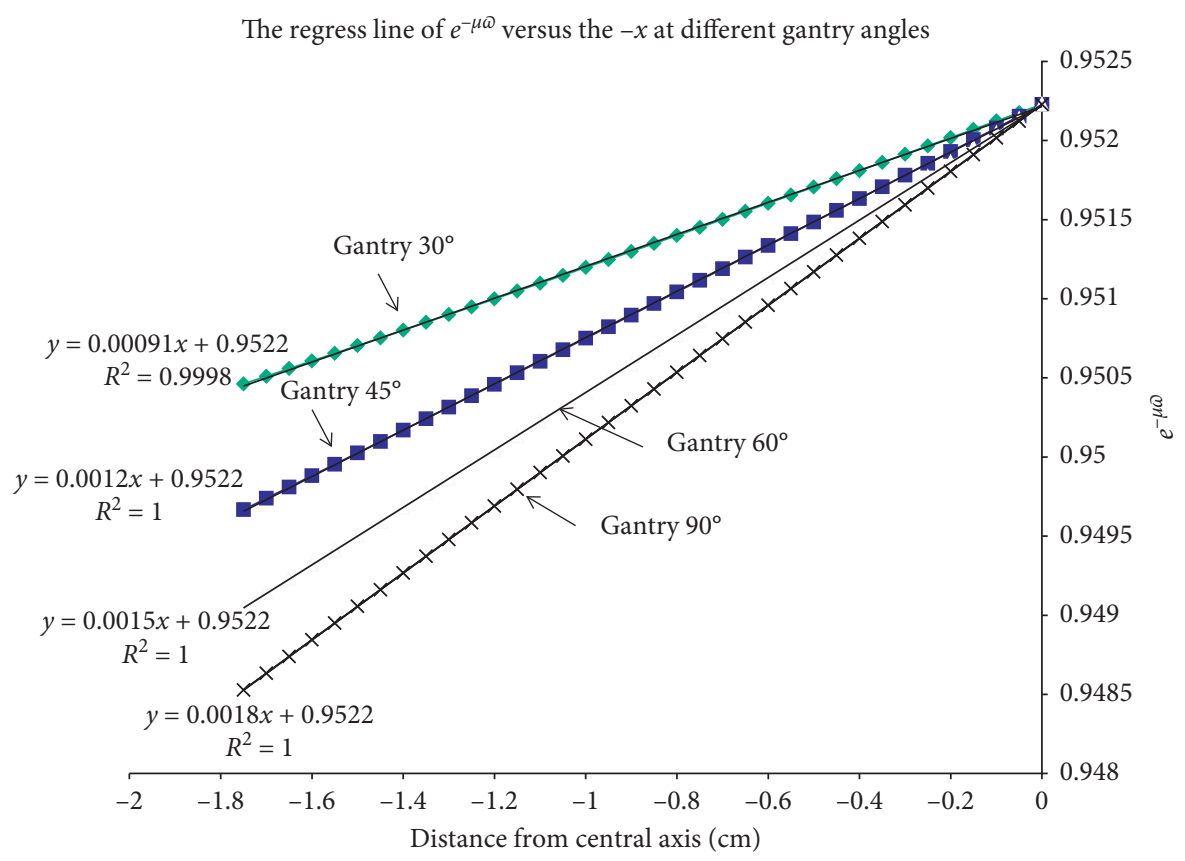

(b)

Figure 7: (a, b) The regression lines of $\mathrm{e}^{-\mu \omega}$, where $\omega=\overline{\mathrm{ed}}$, versus the distance on the $+x$ axis in (a) and the $-x$ axis in (b), at different gantry angles. The slopes are $0.0021,0.0019,0.0015$, and 0.0011 at gantry angles of $90^{\circ}, 60^{\circ}, 45^{\circ}$, and $30^{\circ}$, respectively.

TABle 1: The coplanar penetration depth $\overline{e d}(\omega)$ and $\mathrm{e}^{-\mu \omega}$ on the right-side penumbra after double exposures.

\begin{tabular}{|c|c|c|c|c|c|c|c|c|c|c|}
\hline \multirow{3}{*}{$\begin{array}{l}\text { Distance from central axis of point } d \text { on the } x \text { axis } \\
(\mathrm{cm})\end{array}$} & \multicolumn{10}{|c|}{ Gantry angles } \\
\hline & \multicolumn{2}{|r|}{$0^{\circ}$} & \multicolumn{2}{|c|}{$30^{\circ}$} & \multicolumn{2}{|c|}{$45^{\circ}$} & \multicolumn{2}{|c|}{$60^{\circ}$} & \multicolumn{2}{|c|}{$90^{\circ}$} \\
\hline & $\overline{\mathrm{ed}}(\emptyset)$ & $e^{-\mu \omega}$ & $\overline{\mathrm{ed}}(\omega)$ & $e^{-\mu \omega}$ & $\overline{\mathrm{ed}}(\varpi)$ & $e^{-\mu \omega}$ & $\overline{\mathrm{ed}}(\bowtie)$ & $e^{-\mu \omega}$ & $\overline{\mathrm{ed}}(\omega)$ & $e^{-\mu \omega}$ \\
\hline 0 & 11.00 & $0.95223^{\#}$ & 11.00 & 0.95223 & 11.00 & 0.95223 & 11.00 & 0.95223 & 11.00 & 0.95223 \\
\hline 0.1 & 11.00 & 0.95223 & 10.95 & 0.95233 & 10.93 & 0.95238 & 10.91 & 0.95241 & 10.90 & 0.95244 \\
\hline 0.2 & 11.00 & 0.95223 & 10.90 & 0.95244 & 10.86 & 0.95253 & 10.83 & 0.95260 & 10.80 & 0.95265 \\
\hline 0.3 & 11.00 & 0.95223 & 10.85 & 0.95255 & 10.79 & 0.95268 & 10.74 & 0.95278 & 10.70 & 0.95286 \\
\hline 0.4 & 10.99 & 0.95223 & 10.80 & 0.95266 & 10.71 & 0.95283 & 10.65 & 0.95296 & 10.60 & 0.95308 \\
\hline 0.5 & 10.99 & 0.95223 & 10.74 & 0.95277 & 10.64 & 0.95298 & 10.56 & 0.95315 & 10.50 & 0.95329 \\
\hline 0.6 & 10.99 & 0.95223 & 10.69 & 0.95287 & 10.57 & 0.95313 & 10.48 & 0.95333 & 10.40 & 0.95350 \\
\hline 0.7 & 10.98 & 0.95223 & 10.64 & 0.95298 & 10.50 & 0.95329 & 10.39 & 0.95352 & 10.30 & 0.95371 \\
\hline 0.8 & 10.98 & 0.95223 & 10.58 & 0.95309 & 10.42 & 0.95344 & 10.30 & 0.95370 & 10.20 & 0.95393 \\
\hline 0.9 & 10.97 & 0.95223 & 10.53 & 0.95321 & 10.35 & 0.95359 & 10.21 & 0.95389 & 10.10 & 0.95414 \\
\hline 1 & 10.96 & 0.95223 & 10.47 & 0.95332 & 10.27 & 0.95375 & 10.12 & 0.95407 & 10.00 & 0.95435 \\
\hline 1.1 & 10.96 & 0.95223 & 10.42 & 0.95343 & 10.20 & 0.95390 & 10.04 & 0.95426 & 9.90 & 0.95456 \\
\hline 1.2 & 10.95 & 0.95223 & 10.36 & 0.95354 & 10.13 & 0.95406 & 9.95 & 0.95445 & 9.80 & 0.95477 \\
\hline 1.3 & 10.94 & 0.95223 & 10.30 & 0.95365 & 10.05 & 0.95421 & 9.86 & 0.95463 & 9.70 & 0.95499 \\
\hline 1.4 & 10.93 & 0.95223 & 10.25 & 0.95377 & 9.97 & 0.95437 & 9.77 & 0.95482 & 9.60 & 0.95520 \\
\hline 1.5 & 10.92 & 0.95223 & 10.19 & 0.95388 & 9.90 & 0.95452 & 9.68 & 0.95501 & 9.50 & 0.95541 \\
\hline 1.6 & 10.91 & 0.95223 & 10.13 & 0.95400 & 9.82 & 0.95468 & 9.59 & 0.95519 & 9.40 & 0.95562 \\
\hline 1.7 & 10.90 & 0.95223 & 10.07 & 0.95411 & 9.75 & 0.95483 & 9.50 & 0.95538 & 9.30 & 0.95584 \\
\hline
\end{tabular}

${ }^{\#}$ Data are calculated from $\overline{\mathrm{ed}}(\overline{\mathrm{ed}}=\omega)$ by using $e^{-\mu \omega}, \mu=0.00494 \mathrm{~cm}^{-1}$ at $6 \mathrm{MV}$ in water.

planning systems to model the penumbra dose profiles of megavoltage X-ray beams. However, both functions have the disadvantage of being noncontinuous. For example, the exponential function describing the distribution of penumbra dose profile can be expressed as the edge-spread function (ESF) or the line-spread function (LSF) [11]. According to their mathematical models, the penumbra is fitted by

$$
\begin{aligned}
& \operatorname{ESF}(x)=A e^{\alpha x}+B e^{\beta x}(x \text { at the negative direction }), \\
& \operatorname{ESF}(x)=1-A e^{\alpha x}-B e^{-\beta x}(x \text { at the positive direction }), \\
& \operatorname{LSF}(x)=A \alpha e^{-\alpha|x|}+B \beta e^{-\beta|x|},
\end{aligned}
$$

where $x$ is the distance from the beam edge, namely, center of penumbra, and $A+B=0.5$. 


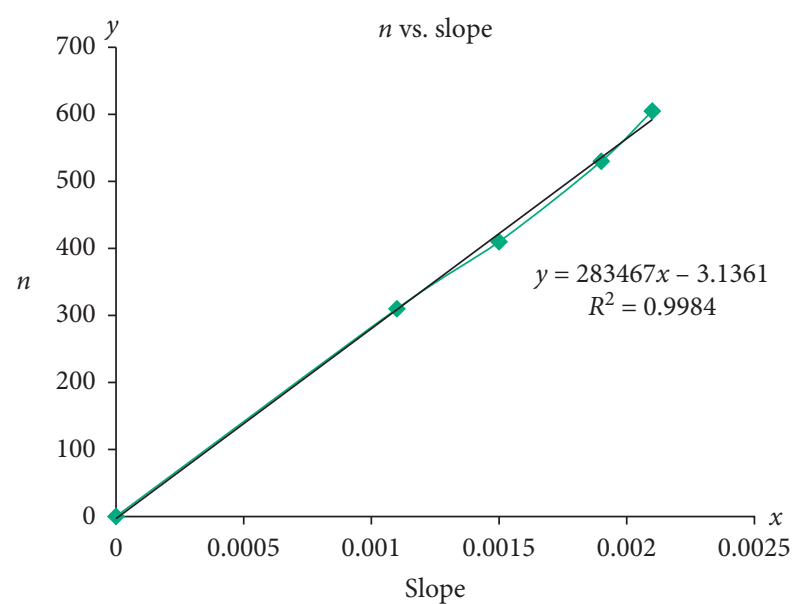

(a)

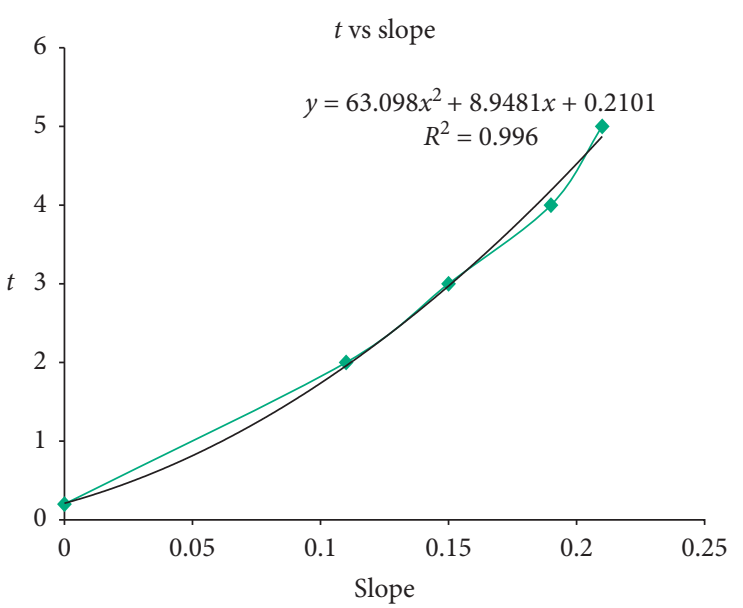

(b)

Figure 8: (a-b) The curves of slope versus $n$ and $t$ were shown in (a,b), respectively. Once the relationships of gantry angle versus slope and slope versus $n$ and $t$ are known, the parameters $n$ and $t$ in the LSRE model can be found easily to fit the measured dose profile or to construct the estimated profile for any gantry angle at coplanar irradiation with good agreement.

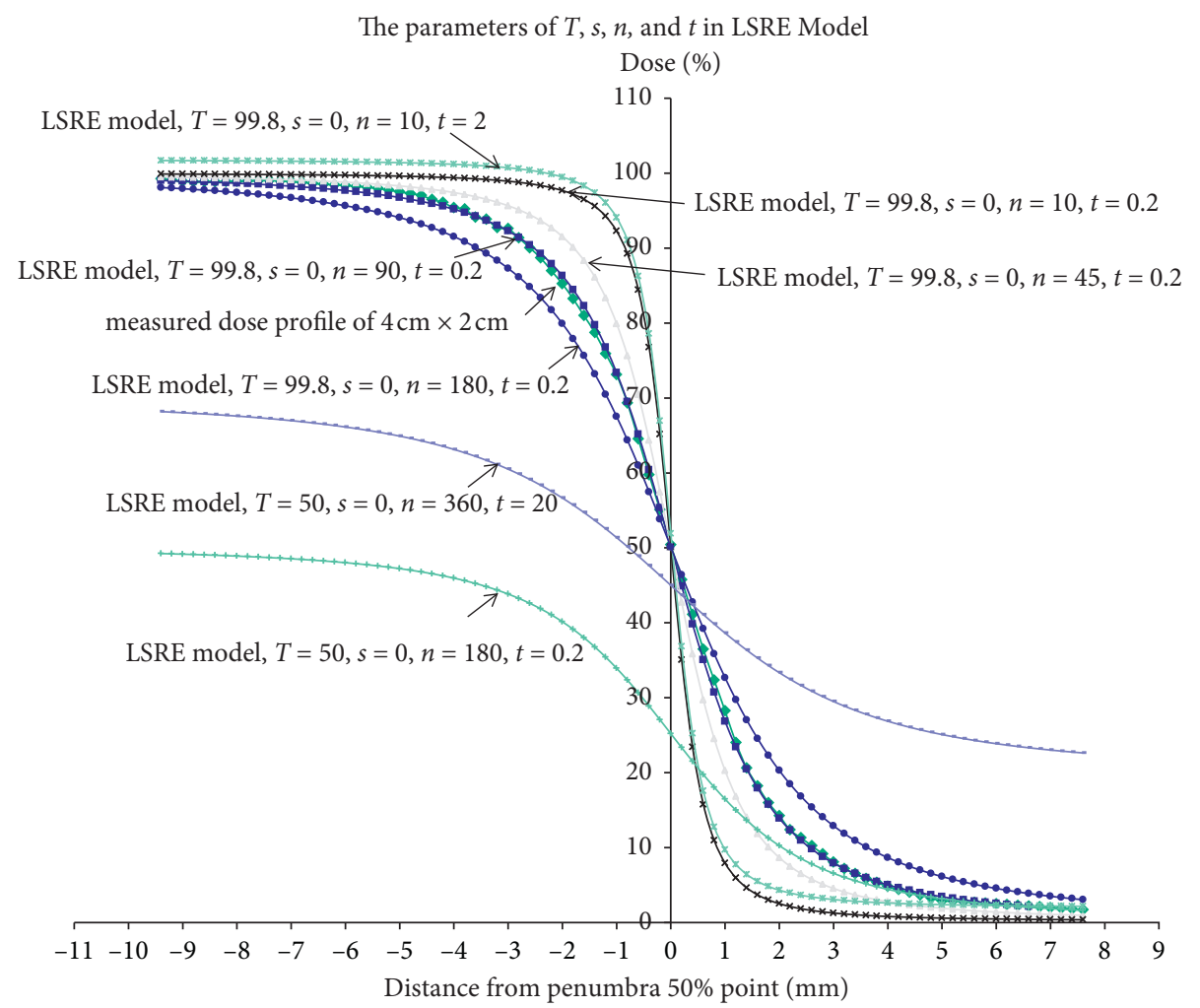

FIGURE 9: The dose profile in the penumbra region on the $x$ axis can be modified by adjusting the $T s, t$, and $n$ in the LSRE model to fit the measured dose profile.

$\mathrm{A}, \mathrm{B}, \boldsymbol{\alpha}$, and $\beta$ are constants. The weak point of this type of penumbra function is the discrete nature in describing the penumbra curve. On the contrary, the LSRE is based on an inverse square root function $[12,13]$ and is a continuous equation that can fit the entire dose profile along the $x$ axis (Figure 9).

Using our model, $n$ and $t$ can be derived easily for the measured and predicted penumbra at any gantry angles once given the gantry angle and then the slope and eventually $n$ and $t$. For example, if we replace $x$ with the slope from Figure $7(\mathrm{a})$ into the equation $y=283467 x-3.1361$, then we get the $y$ value as $n$ (Figure 8(a)); if we replace $x$ with the slope of a known gantry angle from Figure $7(\mathrm{~b})$ into the equation $y=63.098 x^{2}+8.9481 x+0.2101$, then we get the $y$ value as $t$ (Figure $8(\mathrm{~b})$ ). The parameters $n$ and $t$ in the LSRE model can then be used to predict the dose profile with 


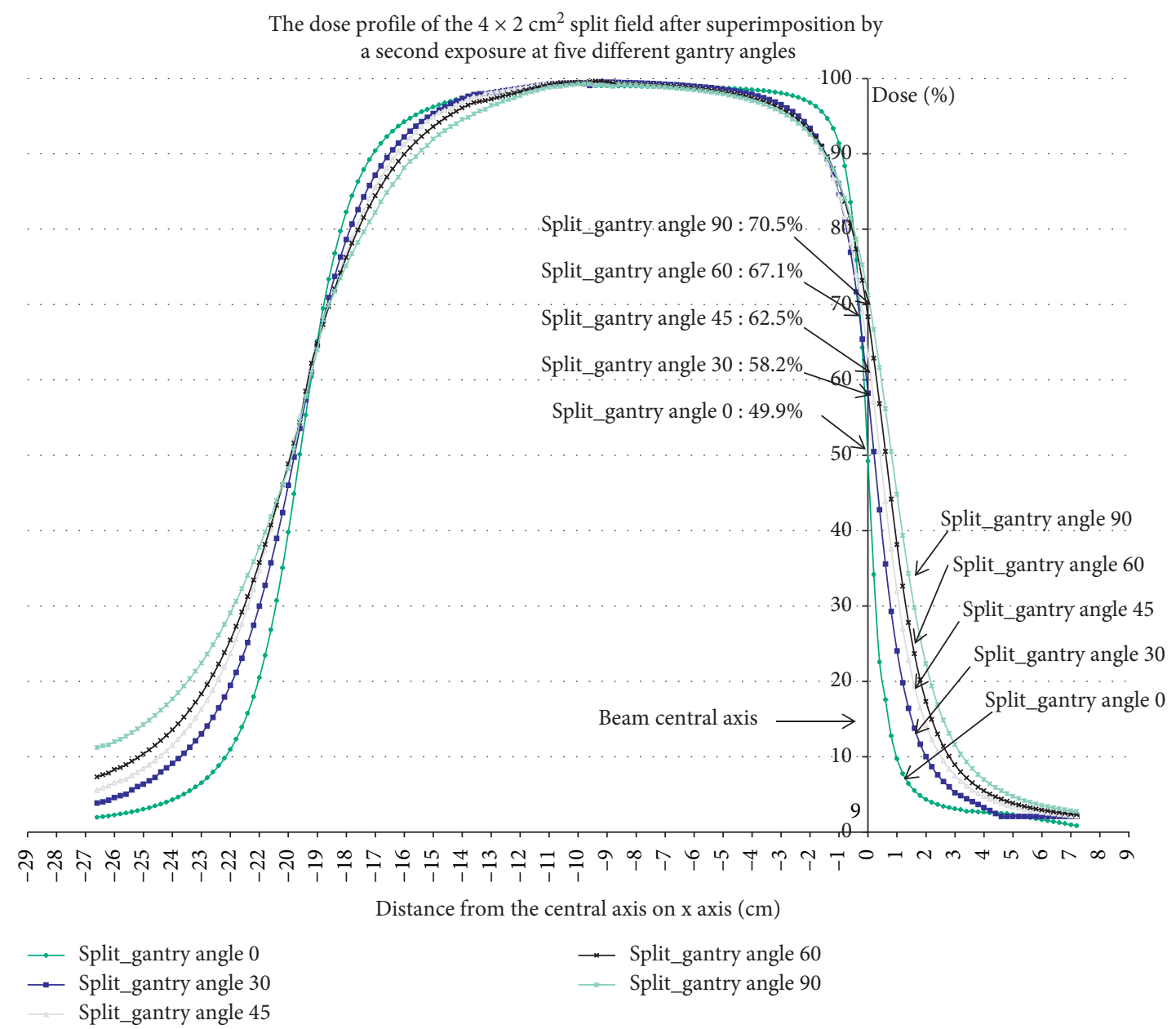

FIGURE 10: The intersection change of the dose profile perturbed by overlapping fields with beam central axis in $4 \times 2 \mathrm{~cm}^{2}$ split field was calculated by LSRE in this study.

precision for any gantry angles at coplanar irradiation. This method enables radiation physicists and treatment planning system programmers to obtain penumbra dose perturbation data by using a mathematical process.

Penumbra region dose data are important in radiotherapy planning systems for monitor unit calculation especially in linear accelerator-based radiosurgery. The precise position of the MLC and the penumbra shape are two important factors for treatment monitor unit calculation. The MLC-related dose penumbra in the treatment planning system must be calibrated to avoid underdosage or overdosage.

For example, the penumbra expansion perturbed by overlapping fields in $4 \times 2 \mathrm{~cm}^{2}$ split field calculated in Figure 10 leads to an intersection change with the standard undisturbed $50 \%$ field edge dose profile of $70.5 \%, 67.1 \%, 62.5 \%$, and $58.2 \%$ at $90^{\circ}, 60^{\circ}, 45^{\circ}$, and $30^{\circ}$ gantry angles, respectively. According to this result, if we ignore tentatively the penumbra position offset correction, the split field monitor unit calculated by the escalated output could result in an overdosage up to $10.5 \%$ in linear accelerator-based radiosurgery technique [14].

Many lung cancer patients who undergo radiation therapy are treated with higher-energy photons such as $10 \mathrm{MV}$ or higher to obtain a deeper penetration and better dose uniformity. However, lower energy such as $6 \mathrm{MV}$ photon beams should be preferred over higher energies photons because of the significant loss of lateral dose equilibrium for high-energy beams in the low-density medium. Any gains in radial dose uniformity across steep density gradients for higher-energy beams must be weighed carefully against the lateral beam degradation due to penumbra widening. The LSRE model can be applied to predict the penumbra perturbation of both highand low-energy photon beams in lesions surrounded by lowdensity organ such as lung.

\section{Conclusion}

Our study shows how to perform an accurate calculation of the penumbra perturbation caused by an overlapping field at different gantry angles by using a mathematical model in linear accelerator-based radiosurgery. The LSRE model is the first continuous equation which describes the dose profile across the entire penumbra. The clinical significance of this study is that the treatment monitor units can be overestimated or underestimated during linear accelerator-based radiosurgery if the penumbra dose profile is not correctly calculated in the treatment planning system. The LSRE model offers a mathematical approach to correct the penumbra and makes it unnecessary to do tedious physics experiments. 


\section{Appendix}

\section{A. The Conceptual Process of LSRE Model}

The LSRE model originated from the proportion function $y(x)=1 / x$. When $x$ increases from $-\infty$ to 0 , the curve of $y$ is at the $(-,-)$ quadrant. When $x$ goes from 0 to $+\infty$, the curve of $y$ is situated at the $(+,+)$ quadrant. Let $1 / x$ become $1 /|x|$; then the curve falls in the $(-,+)$ and the $(+,+)$ quadrants. The curve of $y(x)=1 /|x|$ has a dose-profile-like pattern. Let $y(x)=(1 /|x|)=\left(1 / \sqrt{\left(x^{2}\right)}\right)$. When $x=0, y$ becomes infinite which does not happen in real dose profiles. Therefore, we insert $n$ into $y(x)$ to be $y(x)=\left(1 / \sqrt{n+\left(x^{2}\right)}\right)$, where $n>0$. It avoids the infinite divergence when $x=0$. If we let $y(x)=\left(x / \sqrt{n+\left(x^{2}\right)}\right)$, then $y$ approaches -1 and 1 when $x$ goes from $-\infty$ to $+\infty$, respectively. Furthermore, in order to converge the $y$ value to 1 and 0 when $x$ approaches $-\infty$ and $+\infty$, let $y(\check{s})=(1 / 2)\left(1-\left(\check{s} / \sqrt{\mathrm{n}+\check{s}^{2}}\right)\right)$, where $\check{s}=x-W_{d} / 2$ ( $W_{d} / 2$ is the half-field width, namely, the width from the central axis to the $50 \%$ dose point of the penumbra region). Therefore, $\check{s}=0$ if $x=W_{d} / 2$. The equation $y(\check{s})=(1 / 2)(1-$ $\left.\left(\check{s} / \sqrt{\mathrm{n}+\check{s}^{2}}\right)\right)$ results in $y(\check{s})$ as 1 and $1 / 2$ when $x$ goes from $-\infty$ to the field edge (when $x=W_{d} / 2$, then $\check{s}=0$ and $y(\check{s})=1 / 2$ ) and $y(\check{s})$ as $1 / 2$ and 0 when $x$ goes from the field edge to $+\infty$. Finally, the LSRE model can be expressed as follows:

$$
\text { Penumbra }_{D(\check{s})}=T \cdot \frac{1}{2} \cdot\left(1-\frac{\check{s}}{\sqrt{n+\check{s}^{2}}}\right)+t .
$$

\section{B. The Derivation of Radiation Attenuation Thickness in Ball Phantom}

In Figure 2(a).

$\angle$ bod $=\theta$, where point $b$ is the intersection of the vertical line from point $d$ to $\overline{\mathrm{oa}}$,

$$
\begin{aligned}
\overline{\mathrm{od}} & =\frac{\mathrm{fs}}{2}, \\
\overline{\mathrm{bd}} & =\frac{\mathrm{fs}}{2} \sin (\theta), \\
\beta & =\tan ^{-1}\left(\frac{\overline{\mathrm{bd}} \tan (90-\theta)}{100-\mathrm{bd}}\right) \\
\operatorname{or} \beta & =\tan ^{-1}\left(\frac{\overline{\mathrm{od}} \cos (\theta)}{100}\right), \\
\angle \mathrm{bdc} & =\mathrm{fvc}, \\
\overline{\mathrm{cd}} & =\frac{\overline{\mathrm{bd}}}{\cos (\beta)}, \\
\overline{\mathrm{s}^{\prime} \mathrm{c}} & =\frac{100}{\cos (\beta)},
\end{aligned}
$$

and $\overline{\text { ed }}$ is the penetration depth of the $\overline{S^{\prime}} \mathrm{c}$ from the larger field in the ball phantom. Because $\overline{\mathrm{ed}}=\overline{\mathrm{S}^{\prime} \mathrm{c}}-\overline{\mathrm{cd}}-\overline{\mathrm{S}^{\prime} \mathrm{e}}$, we have to

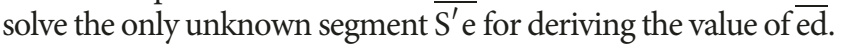

Figure 2(b) is the magnification of the left portion in Figure 2(a). In Figure 2(b), point $e_{(p, q)}$ is the intersection of
$\overline{\mathrm{S}^{\prime} \mathrm{C}}$ with the ball surface. $\overline{\mathrm{S}^{\prime} \mathrm{e}^{2}}=p^{2}+q^{2}, p$ is the distance of $\overline{S^{\prime} f}$, and $q$ is the distance of $\overline{\mathrm{S}^{\prime} \mathrm{e}}$. Point $f$ is the intersection of the horizontal line from point $e$ with $\overline{\mathrm{S}^{\prime} \mathrm{o}}$; in other words, the hinge angle is $90^{\circ}$ of $\overline{\mathrm{S}^{\prime} \mathrm{o}}$ with $\overline{\mathrm{ef}}$.

$\overline{\mathrm{S}^{\prime} \mathrm{e}}$ is derived as follows:

$$
q=\tan \beta \cdot p=\xi p\left(\xi \text { is the slope of } \overline{\mathrm{S}^{\prime} \mathrm{e}}\right) .
$$

$(s-p)^{2}+q^{2}=R^{2}(s=100 \mathrm{~cm}$ and is the source-axis distance which differs from the symbol $s$ used in the LSRE model; distance $R$ is the radius of the ball phantom).

$$
\therefore p^{2}-2 \mathrm{sp}+s^{2}+q^{2}=R^{2} \text {. }
$$

If we substitute $q$ of equation (B.5) with equation (B.3), then

$$
\begin{gathered}
p^{2}-2 s p+s^{2}+\xi^{2} p^{2}=R^{2}, \\
\left(1+\xi^{2}\right) p^{2}-2 s p+s^{2}-R^{2}=0, \\
p=\frac{2 s \pm \sqrt{4 s^{2}-4\left(1+\xi^{2}\right)\left(s^{2}-R^{2}\right)}}{2\left(1+\xi^{2}\right)} .
\end{gathered}
$$

There are two intersection points of ray line $\overline{\mathrm{S}^{\prime} \mathrm{e}^{\prime}}$ with the ball phantom surface which can be expressed as $2 s+\sqrt{4 s^{2}-4\left(1+\xi^{2}\right)\left(s^{2}-R^{2}\right)} / 2\left(1+\xi^{2}\right)$ (point $\left.e^{\prime}\right)$ and $2 s-$ $\sqrt{4 s^{2}-4\left(1+\xi^{2}\right)\left(s^{2}-R^{2}\right)} / 2\left(1+\xi^{2}\right) \quad($ point $e)$ or as $2 s \pm \sqrt{4 s^{2}-4\left(1+\xi^{2}\right)\left(s^{2}-R^{2}\right)} / 2\left(1+\xi^{2}\right)$ for the 2 points of $e$ and $e^{\prime}$. Point $e$ is near $S^{\prime}$, while point $e^{\prime}$ is far from $S^{\prime}$ (Figure 2(b)). We choose point $e$, namely, $2 s-\sqrt{4 s^{2}-4\left(1+\xi^{2}\right)\left(s^{2}-R^{2}\right)}$ for the calculation of $p$ :

$$
\therefore p=\frac{2 s-\sqrt{4 s^{2}-4\left(1+\xi^{2}\right)\left(s^{2}-R^{2}\right)}}{2\left(1+\xi^{2}\right)} \text {. }
$$

Since $q=\xi p$,

$\therefore \overline{S^{\prime} \mathrm{e}}=\sqrt{p^{2}+q^{2}}=\sqrt{\left(1+\xi^{2}\right) p^{2}}=\frac{s-\sqrt{s^{2}-\left(1+\xi^{2}\right)\left(s^{2}-R^{2}\right)}}{\sqrt{\left(1+\xi^{2}\right)}}$.

Once $\overline{\mathrm{cd}}, \overline{\mathrm{S}^{\prime} \mathrm{c}}$, and $\overline{\mathrm{S}^{\prime} \mathrm{e}}$ are derived, then $\overline{\mathrm{ed}}$ can be obtained:

$$
\overline{\mathrm{ed}}=\overline{\mathrm{S}^{\prime} \mathrm{c}}-\overline{\mathrm{cd}}-\overline{\mathrm{S}^{\prime} \mathrm{e}}
$$

\section{Data Availability}

The GAF chromic films and manipulation process data used to support the findings of this study were supplied by Jia-Ming Wu under license and so cannot be made freely available. Requests for access to these data should be made to Jia-Ming Wu, Ph.D., Department of Radiation Oncology, Yee Zen General Hospital, \#30, Alley 321, Yuan Xin North Road, Tao Yuan, Taiwan (e-mail: jiaming.wu@ chmsc.com). 


\section{Conflicts of Interest}

There are no conflicts of interest.

\section{Authors' Contributions}

Shi-Qiang Tang, Yee-Min Jen, and Jia-Ming Wu contributed equally to this work.

\section{Acknowledgments}

This study was supported by a grant from the Chenzhou No.1 People's Hospital (Grant no. N2014-016).

\section{References}

[1] B. Fraass, K. Doppke, M. Hunt et al., "American association of physicists in medicine radiation therapy committee task group 53: quality assurance for clinical radiotherapy treatment planning," Medical Physics, vol. 25, no. 10, pp. 1773-1829, 1998.

[2] F. Chen, J.-W. Shi, Z.-S. Yin, Y. Peng, Y.-M. Jen, and J.-M. Wu, "Dose distribution characteristic study of the rotating gamma knife (RGK) by using the geometry analytic method," Medical Dosimetry, vol. 42, no. 4, pp. 251-267, 2017.

[3] H. Beauvais, A. Bridier, and A. Dutreix, "Characteristics of contamination electrons in high energy photon beams," Radiotherapy and Oncology, vol. 29, no. 3, pp. 308-316, 1993.

[4] T. Kamomae, Y. Miyabe, A. Sawada et al., "Simulation for improvement of system sensitivity of radiochromic film dosimetry with different band-pass filters and scanner light intensities," Radiological Physics and Technology, vol. 4, no. 2, pp. 140-147, 2011.

[5] R. Dreindl, D. Georg, and M. Stock, "Radiochromic film dosimetry: considerations on precision and accuracy for EBT2 and EBT3 type films," Medical Physics, vol. 24, no. 2, pp. 153-163, 2014.

[6] D. Slobodan, T. Nada, and L. David, "Reference radiochromic film dosimetry: review of technical aspects," Physica Medica, vol. 32, no. 4, pp. 541-556, 2016.

[7] O. A. García-Garduño, J. M. Lárraga-Gutiérrez, M. Rodríguez-Villafuerte, A. Martínez-Dávalos, and T. Rivera-Montalvo, "Effect of correction methods of radiochromic EBT2 films on the accuracy of IMRT QA," Applied Radiation and Isotopes, vol. 107, pp. 121-126, 2016.

[8] M. Raphan and E. P. Simoncelli, "Least squares estimation without priors or supervision," Neural Computation, vol. 23, no. 2, pp. 374-420, 2011.

[9] B. Vanderstraeten, N. Reynaert, L. Paelinck et al., "Accuracy of patient dose calculation for lung IMRT: a comparison of Monte Carlo, convolution/superposition, and pencil beam computations," Medical Physics, vol. 33, no. 9, pp. 3149-3158, 2006.

[10] H. Keivan, D. Shahbazi-Gahrouei, A. Shanei, and A. Amouheidari, "Assessment of imprecise small photon beam modeling by two treatment planning system Algorithms," Journal of Medical Signals \& Sensors, vol. 8, no. 1, pp. 39-45, 2018.

[11] M. J. Day, G. D. Lambert, and S. M. Locks, "The effect of secondary electron spread on the penumbra in high energy photon beam therapy," The British Journal of Radiology, vol. 63 , no. 748 , pp. $278-285,1990$.

[12] S. C. T. Choi and M. A. Saunders, "Algorithm 937: MINRESQLP for symmetric and hermitian linear equations and Least- squares problems," ACM Transactions on Mathematical Software, vol. 40, no. 2, pp. 1-12, 2014.

[13] P. Tavallali, M. Razavi, and S. Brady, "A non-linear data mining parameter selection algorithm for continuous variables," PLoS One, vol. 12, no. 11, Article ID e0187676, 2017.

[14] J. M. Wu, T. F. Lee, and C. M. Kuo, "A light field-based method to adjust rounded leaf end MLC position for split shape dose calculation correction in a radiation therapy treatment planning system," Journal of Applied Clinical Medical Physics, vol. 13, no. 6, pp. 3-18, 2012. 


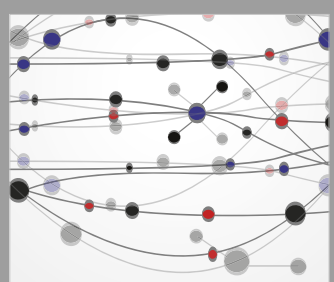

The Scientific World Journal
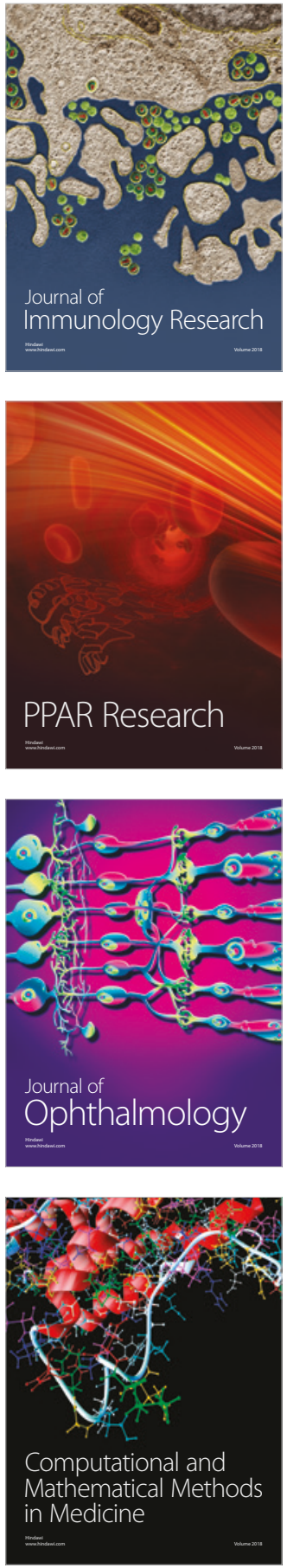

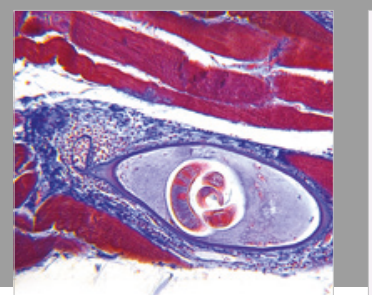

Gastroenterology Research and Practice

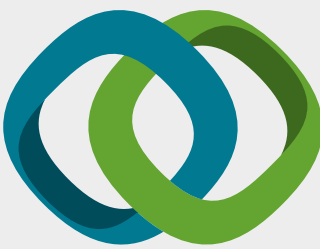

\section{Hindawi}

Submit your manuscripts at

www.hindawi.com
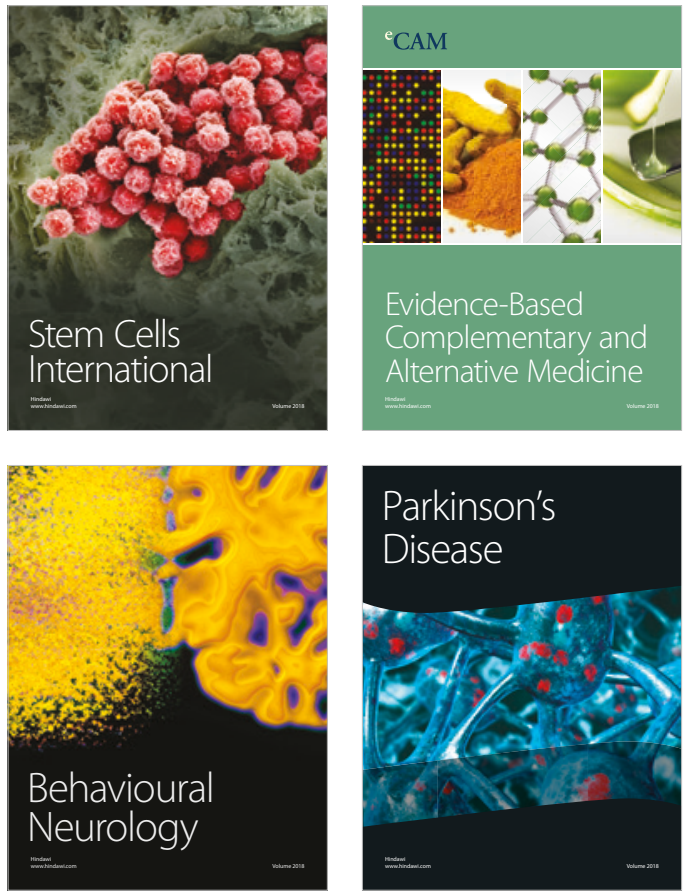

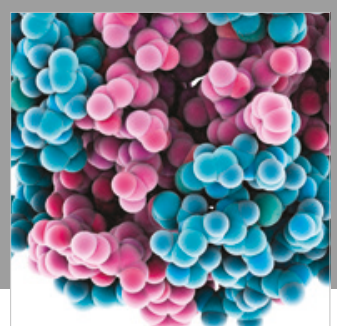

ournal of

Diabetes Research

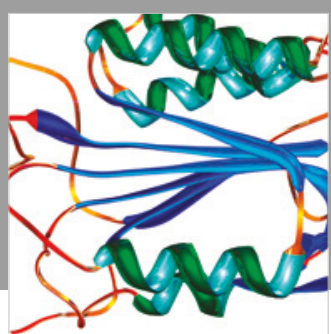

Disease Markers
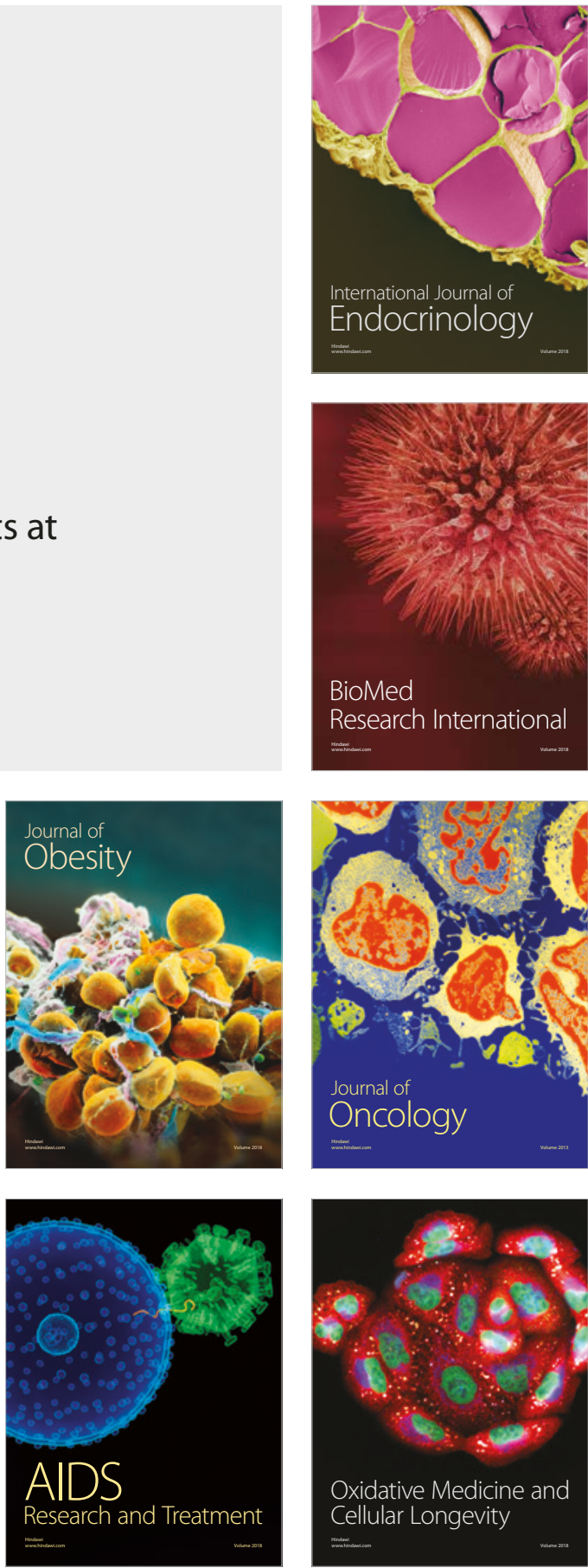